\title{
Effect of Characteristic Spectral Lines on Rock Identification of LIBS
}

\author{
Ke ZhiQuan ${ }^{1}$, Wang YangEn ${ }^{1,2^{*}}, \mathrm{Xu} \mathrm{Yi}^{1}$, Dong $\mathrm{XiPu}^{1}$ and Zhou MaoHui ${ }^{1}$ \\ 1.School of Physics and Optoelectronic Engineering, Yangtze University, Jingzhou 434023, China \\ 2. Beijing Key Laboratory of Optical Detection Technology for Oil and Gas, China University of Petroleum, Beijing 102249, China
}

\begin{abstract}
The LIBS (Laser induced-breakdown spectroscopy) combined with BPNN (Back propagation neural network) was applied in rock sorting and distinguishing for 26 rock samples of 6 types. According to contents of major elements in samples, we selected lines of $\mathrm{Si}, \mathrm{Al}, \mathrm{Fe}, \mathrm{K}, \mathrm{Ca}, \mathrm{Mg}, \mathrm{Na}$, Ti and $\mathrm{Mn}$. These lines of 9 elements composed three characteristic spectral models which were the WSLM (Wide spectral line model), the PM (Peak model) and the PRM (Peak ratio model). The first and the second characteristic spectral model were divided into 9 kinds, as follows: the characteristic spectrum with 1 element, the characteristic spectrum with 2 elements, we can deduce the rest from this and the last one has 9 elements. The third model was divided into 8 kinds which were using $\mathrm{Al}$ as reference element. We analysed spectrums of the three models by BPNN. Experimental results shown that whether sorting or distinguishing these samples, identification accuracies of the PM were more than that of the PRM overall, the same as the WSLM did to the PM. While the selected number of elements was 5, 6 or 7, the identification accuracy of the WSLM could reach more than $90 \%$. Continuing to add the number of elements to improve identification accuracy was not very obvious.
\end{abstract}

Key words: LIBS (Laser induced-breakdown spectroscopy), BPNN (Back propagation neural network), characteristic spectral model, WSLM (Wide spectral line model).

\section{Introduction}

LIBS (Laser induced-breakdown spectroscopy) as a measurement technique, based on atomic emission spectroscopy and laser plasma emission spectroscopy, is used to obtain components and contents of material. Having the advantages of fast analysis, all element detecting, remote non-contact testing, ect., this technology is widely used in environmental monitoring [1, 2], historical relics identification [3, 4], biology and medicine $[5,6]$, and food security $[7,8]$. LIBS appears even in space exploration field, the typical symbol is that the United States (U.S.) launched Curiosity rover to Mars in 2012. It carries the ChemCam instrument, which is shooting its laser daily to help us understand whether rocks and soils on Mars have been altered by water and contain chemicals necessary for life $[9,10]$.

\footnotetext{
*Corresponding author: Wang YangEn, MSc, professor, research field: spectral analysis. E-mail: yewang@yangtzeu.edu.cn.
}

The behavior of LIBS laser ablates the samples is very complex, such as laser wavelength, laser energy, material physical and chemical properties, and ambient gas composition and pressure all will directly affect the final spectral profile. In the spectroanalysis of lithology classification or identification, we need some data processing methods. First, the internal standard method, external standard method and calibration-free method are usually used to establish quantitative analysis standards; Second, we require the PIM (Peak intensity model), the NIM (Normalized intensity model), the RIM (Ratio of intensity model) and other characteristic models for reducing the amount of data; Third, writing or directly using algorithms from the software tools to process data and obtain the analysis results is necessary, algorithms are PCA (Principal component analysis) [11], ANN (Artificial neural network) [12], PLS (Partial least squares) [13], SVM (Support vector machine) [14], ect. These three processes to some extent can reduce 
the noise in spectrums, redundan information, matrix effect and other interference and can improve the validity and accuracy of calculation results. Wang Chao [15] took LIBS technology for the analysis of solid samples, he had achieved quantitative detection of elements components and contents of mineral samples and obtained precise identification of lithology. Zhu YiXiang [16] respectively used MRA (Multiple regression analysis), ANN and SVM to realize the lithology identification of 9 volcanic rocks. The results shown that SVM was suitable for lithology identification of volcanic rocks, of which the nonlinear relationship between volcanic lithology and petrophysical parameters was very strong.

According to contents of major elements in samples, we selected 9 elements lines from the LIBS spectra to sort and distinguish rock samples. Then we established three feature spectrums models, which were the WSLM, the PM, and the PRM. After the data of these three models were trained and predicted by BPNN (Back propagation neural network), we obtained the model with the highest identification accuracy by comparing identification accuracies of them.

\section{Experimental Setup}

Experimental apparatus, RT100-HP LIBS, is made by Applied Spectra, Inc. ASI. The laser source of the instrument is a Q-switched Nd: YAG laser operating at the wavelength of $1,064 \mathrm{~nm}$. The width of laser pulse is less than $5 \mathrm{~ns}$, and the maximum energy of single pulse is $51 \mathrm{~mJ}$. This apparatus has the function of laser energy modulation, and can focus laser with the focal size range from 35 to $200 \mu \mathrm{m}$. A CMOS (Complementary metal-oxide semiconductor) color camera for image displaying of sample surface installed in the apparatus, which is equipped with 670 $\mathrm{nm}$ red laser for guiding ablation point. Using double grating Czerny-Turner spectrometer and ICCD (Intensified charge coupled device) detector to collect spectral signals, this system is also inbuilt gate delay and gate width controller to adjust spectral signals. And it has a built-in spectral analysis software named Axiom and a True LIBS database of emission spectrum.

Set up parameters for testing samples as follows: laser energy was $10 \mathrm{~mJ}$, diameter of laser spot was $200 \mu \mathrm{m}$, gate width was $3 \mu \mathrm{s}$, gate delay was $2 \mu$ s and frequency of repetition was $4 \mathrm{~Hz}$.

In laboratory, the apparatus used to squeeze samples was 769YP-30T, of which the maximum pressure can reach $35 \mathrm{MPa}$. The samples were squeezed for $20 \mathrm{~min}$ by a pressure of $30 \mathrm{MPa}$, then they were analysed by laser induced-breakdown spectrometer in the sample chamber. Each sample was analysed on two points apart from $1 \mathrm{~mm}$, and measured 10 times on each point.

\section{Spectral Lines Models}

The 26 rock samples with different components and contents of elements were divided into six categories: Black Mudstone, Gray-Black Mudstone, Dark-Gray Mudstone, Gray-Black Lime Mudstone, Gray-Black Silty Mudstone and Dark-Gray Lime Mudstone. The main elements of different samples are basically the same, it is very difficult to distinguish different lithology through categories of elements. But contents of elements in rocks make a difference, because of that we can distinguish lithology. According to contents of main elements in samples, 9 main elements were selected: $\mathrm{Si}, \mathrm{Al}, \mathrm{Fe}, \mathrm{K}, \mathrm{Ca}, \mathrm{Mg}, \mathrm{Na}, \mathrm{Ti}$ and $\mathrm{Mn}$. While contents of 9 major elements in samples were more than $100 \mathrm{ppm}$, we selected a peak for every element as the research object. The selection principle of each peak was no obvious self-absorption, well profile, small disturbance and high SNR (Signal-to-noise ratio) [17]. Based on the selected spectral lines, three spectral models were established: the WSLM, the PM and the PRM.

\subsection{Wide Spectral Line Model (WSLM)}

WSLM: These lines of 9 elements constituted the 
characteristic spectra, the characteristic spectrum of each element was a set of spectral lines, of which two sides were symmetric distribution. And wavelengths of peaks of characteristic spectra were center wavelength. The selected elements, center wavelength, wavelength range, spectral lines number and raster number were shown in Table 1.

Nine (9) elements of Sample 1, Sample 2 and Sample 3 chosen from 26 rock samples consisted of the WSLM as shown in Fig. 1. So they were only part of the WSLM.

\subsection{Peak Model (PM)}

PM: These lines of 9 elements constituted the characteristic spectra, the characteristic spectrum of each element was consisted of one spectral line, of which the wavelength was the center wavelength. The chosen spectral lines in the PM were the spectral lines corresponding to the center wavelength in the WSLM.

Nine (9) elements of Sample 1, Sample 2 and Sample 3 chosen the same as the WSLM consisted of the PM as shown in Fig. 2.

\subsection{Peak Ratio Model (PRM)}

PRM: We selected one element from 9 elements as the reference element. These ratios of peak intensity of the other elements to that of reference element constituted the characteristic spectra. In this paper, $\mathrm{Al}$ was chosen as the reference element in the PRM for the following reasons:

Table 1 Selected analysis lines of LIBS spectra of samples.

\begin{tabular}{lllll}
\hline Elements & Center wavelength $(\mathrm{nm})$ & Wavelength range $(\mathrm{nm})$ & Spectral lines number & Raster number $(\mathrm{mm})$ \\
\hline $\mathrm{Mn}$ & 279.482 & $279.121-279.824$ & 15 & 2,400 \\
$\mathrm{Mg}$ & 285.213 & $284.895-285.583$ & 15 & 2,400 \\
$\mathrm{Si}$ & 288.158 & $287.831-288.511$ & 15 & 2,400 \\
$\mathrm{Fe}$ & 301.898 & $301.595-302.232$ & 15 & 2,400 \\
$\mathrm{Al}$ & 309.271 & $308.923-309.594$ & 15 & 2,400 \\
$\mathrm{Ti}$ & 365.350 & $365.056-365.668$ & 15 & 2,400 \\
$\mathrm{Ca}$ & 393.367 & $393.067-393.647$ & 15 & 2,400 \\
$\mathrm{Na}$ & 588.995 & $588.382-589.755$ & 15 & 1,200 \\
$\mathrm{~K}$ & 766.490 & $765.930-767.103$ & 15 & 1,200 \\
\hline
\end{tabular}

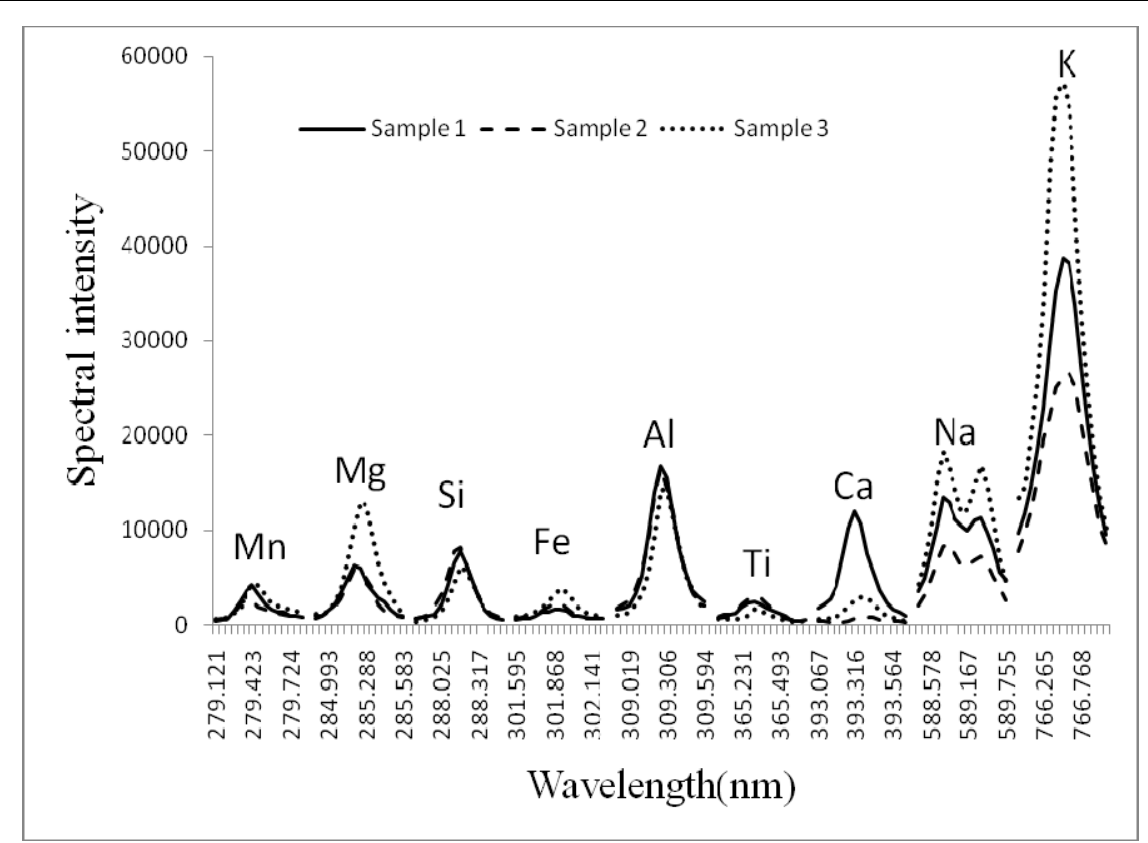

Fig. 1 Nine (9) elements of three rock samples consisted of the WSLM. 
Suppose that Al has the same volatile property, diffusion property and ionization degree as the other 8 elements, then the ratio of intensity of the other elements to that of Al by Eq. (1) [18]:

$$
R=B e^{-\frac{E-E_{0}}{k_{B} T}} \frac{C}{C_{0}}
$$

where, $B$ is constant; $C$ is the contents of the other elements in the sample; $C_{0}$ is the contents of $\mathrm{Al}$ in the sample; $E$ is the excitation energy of the other elements; $E_{0}$ is the excitation energy of $\mathrm{Al} ; k_{B}$ is Boltzmann constant; and $T$ is the excitation temperature, that is, it is the temperature of plasma. From Eq. (1), we know that the closer the excitation energy of the element or the wavelength with $\mathrm{Al}$ is, the smaller the influence of the plasma temperature on the peak ratio of spectral lines is. The center wavelength of $\mathrm{Al}$ was between the maximum and the minimum center wavelength of 9 selected elements, and the center wavelength of $\mathrm{Al}$ was close to others. So that the peak ratios of spectral lines were less affected by the plasma temperature.

The peak intensity of Al was relatively high in 9 selected elements and had well profile, small interference and high signal-to-noise ratio, so that it was less affected by noise and other elements spectrum. The above point can refer to Fig. 1.
Nine (9) elements of Sample 1, Sample 2 and Sample 3 chosen the same as the WSLM consisted of the PRM shown in Fig. 3.

\section{Results and Discussion}

BPNN is a feed-forward neural network, of which it adjust the connection weight by using back propagation learning algorithm. This algorithm based on the principle of error gradient descent designed for adjusting the network weights and threshold, it is in order to realize the minimum of training error [19]. In this paper, BPNN was realized by Matlab, and wavelengths of elements were the input variable. The 20 groups spectral data of each sample were equally divided into two groups. One group was trained by BPNN, and another was identified by BPNN.

\subsection{Identification Accuracy of BPNN in the WSLM}

In the WSLM, one (1) element was selected from 9 elements, the identification accuracy of the BPNN was shown in Table 2, and identification accuracies were reordered by small to large. while selecting the same number of elements with different types, the identification accuracy of samples was not the same, Tables 2-4 can illustrate this point.

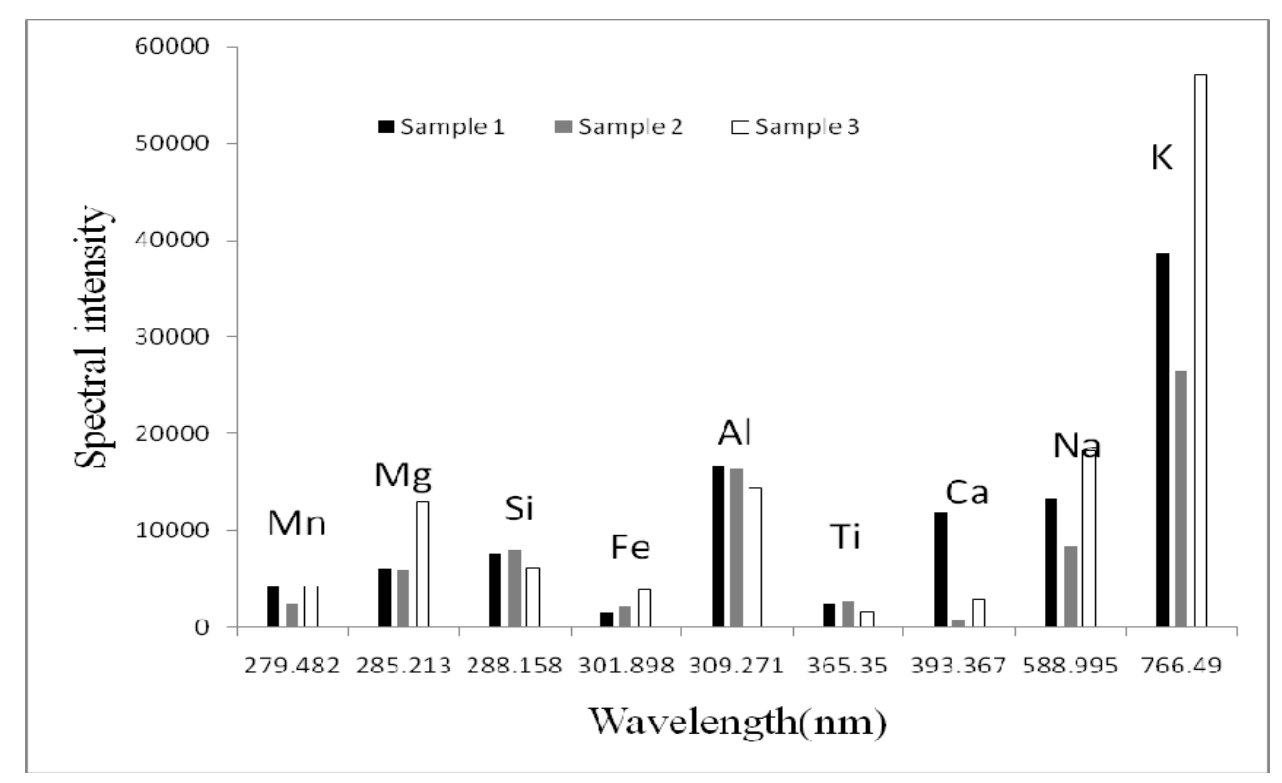

Fig. 2 Nine (9) elements of three rock samples consisted of the PM. 


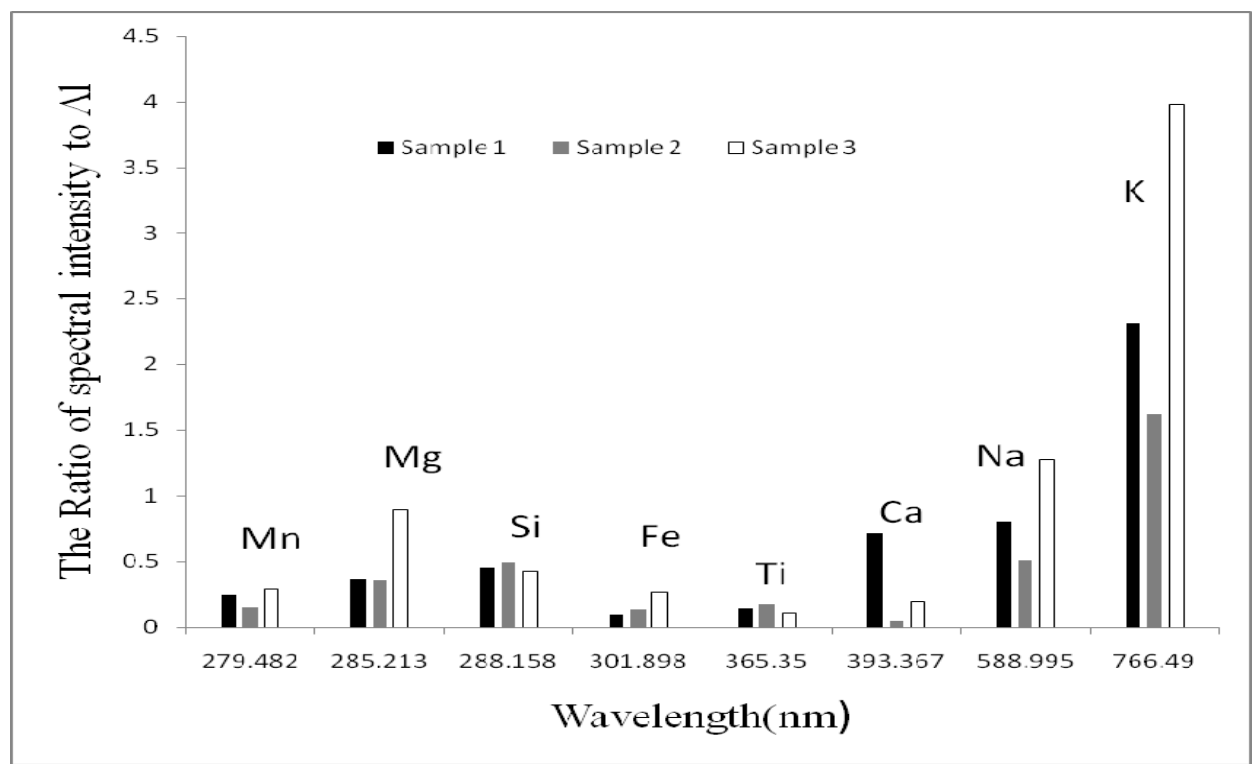

Fig. 3 Nine (9) elements of three rock samples consisted of the PRM.

Table 2 In the WSLM, one element was selected from 9 elements for BPNN analysis.

\begin{tabular}{lllll}
\hline \multirow{2}{*}{ Serial No. } & \multicolumn{2}{c}{ The classification of 6 types of samples } & \multicolumn{2}{c}{ The distinction of 26 samples } \\
\cline { 2 - 5 } & Element & Identification accuracy (\%) & Element & Identification accuracy (\%) \\
\hline 1 & $\mathrm{Fe}$ & 40.15 & $\mathrm{Fe}$ & 25.19 \\
2 & $\mathrm{Mn}$ & 43.73 & $\mathrm{Mn}$ & 27.46 \\
3 & $\mathrm{Mg}$ & 48.12 & $\mathrm{Mg}$ & 30.92 \\
4 & $\mathrm{Ti}$ & 53.96 & $\mathrm{Na}$ & 33.42 \\
5 & $\mathrm{Si}$ & 54.00 & $\mathrm{Al}$ & 34.62 \\
6 & $\mathrm{Na}$ & $\mathrm{Ca}$ & 35.46 \\
7 & $\mathrm{Al}$ & 54.81 & $\mathrm{Si}$ & 37.15 \\
8 & $\mathrm{~K}$ & $\mathrm{Ti}$ & 37.27 \\
9 & $\mathrm{Ca}$ & 59.96 & $\mathrm{~K}$ & 51.88 \\
\hline
\end{tabular}

In the WSLM, eight (8) elements was selected from 9 elements for BPNN analysis, these results of identification accuracy were shown in Table 3, of which identification accuracies were sequenced by small to large. While Ti was removed from 9 elements, we selected 7 elements from the remained for BPNN analysis, identification accuracies were shown in Table 4, of which identification accuracies were sequenced by small to large too. In Tables 3 and 4, "8-\#" meant the element of "\#" was removed from 9 elements, and remains were for combination. E.g. "8-K" meant $\mathrm{K}$ was removed from $\mathrm{Si}, \mathrm{Al}, \mathrm{Fe}, \mathrm{K}, \mathrm{Ca}$, $\mathrm{Mg}, \mathrm{Na}, \mathrm{Ti}$ and $\mathrm{Mn}$, and remains were for combination.

From Table 3, we can see that the biggest identification accuracy was the combination of 9 elements except $\mathrm{Ti}$ in the classification of 6 types of samples, and the biggest identification accuracy was the combination of 9 elements except $\mathrm{Mn}$ in the distinction of 26 samples. From Table 4, as can be seen that the biggest identification accuracy was the combination of 8 elements except $\mathrm{Al}$ in the classification of 6 types of samples, and the biggest identification accuracy was the combination of 8 elements except $\mathrm{Mg}$ in the distinction of 26 samples. These shown that when we chose 7 or 8 elements from 9 elements to combine, the bigger identification accuracy would be the combination of elements of which identification accuracies were higher in Table 2. For the combination of 2 to 6 elements, the maximum 
Table 3 In the WSLM, 8 elements was selected from 9 elements for BPNN analysis.

\begin{tabular}{llllc}
\hline \multirow{2}{*}{ Serial No. } & \multicolumn{3}{c}{ The classification of 6 types of samples } & The distinction of 26 samples \\
\cline { 2 - 5 } & Element & Identification accuracy (\%) & Element & Identification accuracy (\%) \\
\hline 1 & $8-\mathrm{K}$ & 87.96 & $8-\mathrm{K}$ & 88.77 \\
2 & $8-\mathrm{Al}$ & 89.65 & $8-\mathrm{Ca}$ & 90.81 \\
3 & $8-\mathrm{Ca}$ & 90.04 & $8-\mathrm{Si}$ & 92.58 \\
4 & $8-\mathrm{Fe}$ & 90.08 & $8-\mathrm{Na}$ & 92.92 \\
5 & $8-\mathrm{Si}$ & 90.50 & $8-\mathrm{Ti}$ & 94.27 \\
6 & $8-\mathrm{Mg}$ & 91.08 & $8-\mathrm{Mg}$ & 94.31 \\
7 & $8-\mathrm{Mn}$ & 91.12 & $8-\mathrm{Al}$ & 94.46 \\
8 & $8-\mathrm{Na}$ & 91.12 & $8-\mathrm{Fe}$ & 94.69 \\
9 & $8-\mathrm{Ti}$ & 91.96 & $8-\mathrm{Mn}$ & 95.35 \\
\hline
\end{tabular}

Table 4 In the WSLM, 7 elements was selected from 8 elements for BPNN analysis.

\begin{tabular}{llllc}
\hline \multirow{2}{*}{ Serial N0. } & \multicolumn{3}{c}{ The classification of 6 types of samples } & The distinction of 26 samples \\
\cline { 2 - 5 } & Element & Identification accuracy (\%) & Element & Identification accuracy (\%) \\
\hline 1 & $7-\mathrm{Ca}$ & 86.35 & $7-\mathrm{K}$ & 83.15 \\
2 & $7-\mathrm{K}$ & 86.46 & $7-\mathrm{Ca}$ & 88.58 \\
3 & $7-\mathrm{Na}$ & 89.42 & $7-\mathrm{Na}$ & 91.42 \\
4 & $7-\mathrm{Mn}$ & 90.00 & $7-\mathrm{Si}$ & 92.73 \\
5 & $7-\mathrm{Si}$ & 90.04 & $7-\mathrm{Mn}$ & 92.88 \\
6 & $7-\mathrm{Fe}$ & 90.15 & $7-\mathrm{Fe}$ & 93.65 \\
7 & $7-\mathrm{Mg}$ & 90.42 & $7-\mathrm{Al}$ & 94.08 \\
8 & $7-\mathrm{Al}$ & 91.04 & $7-\mathrm{Mg}$ & 94.35 \\
\hline
\end{tabular}

identification accuracy was also consistent with the above rule, which also applied to the PM and the PRM. In Table 2, identification accuracies of $\mathrm{Ti}, \mathrm{Mn}, \mathrm{Al}$ and $\mathrm{Mg}$ were not the lowest, it follows that the combination with the maximum identification accuracy was not consisted by the elements except the element with the smallest identification accuracy in Table 2. The reason for this is that the combination of different elements could improve or decrease the BPNN identification accuracy.

\subsection{The Effect of 9 Elements on the Identification Accuracy of BPNN Respectively.}

Selecting 1 element from 9 elements to constitute three characteristic spectra which were input to BPNN to sort or distinguish samples. The effect of 9 elements on the identification accuracy of BPNN respectively was shown in Fig. 4, and identification accuracies of the WSLM can refer to Table 2. And Model 1 represented the WSLM, Model 2 represented the PM and Model 3 represented the PRM. This definition is general in this paper. Because of the $\mathrm{Al}$ was used as the reference element in Model 3, so there were no identification accuracies of Al in Fig. 4.

From Fig. 4, it can be seen that identification accuracies of samples were not much high, and the maximum was no more than $65 \%$. Whatever elements except Fe were selected, identification accuracies of Model 1 were higher than that of the other two models, of which identification accuracies were very close. In the classification of 6 types of samples, we chose Mn, $\mathrm{Si}, \mathrm{Fe}, \mathrm{K}$, or $\mathrm{Mg}$, identification accuracies of Model 2 were higher than that of Model 3. However, when the 26 samples were distinguished, whatever element we chose, the above conclusion still held water. It can also be seen that identification accuracies of BPNN in the three characteristic models had some similarity with the change of elements.

Three characteristic models had part of the same spectral lines which meant the center spectral lines of 


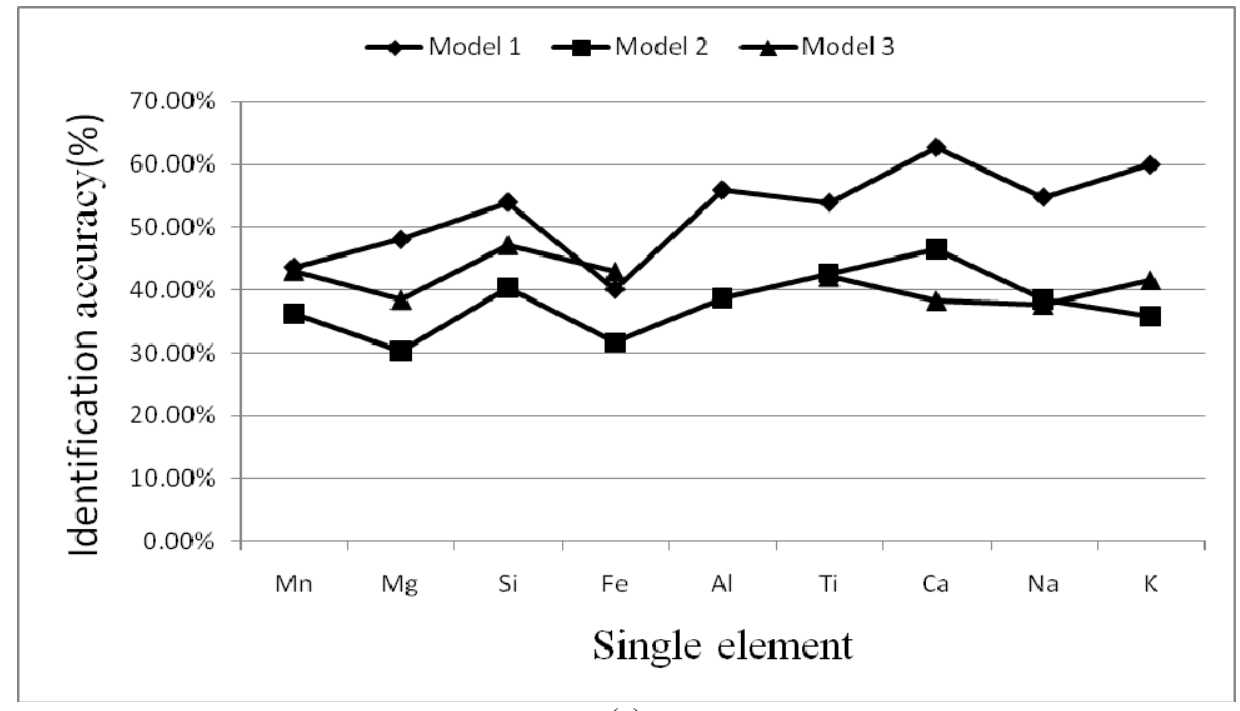

(a)

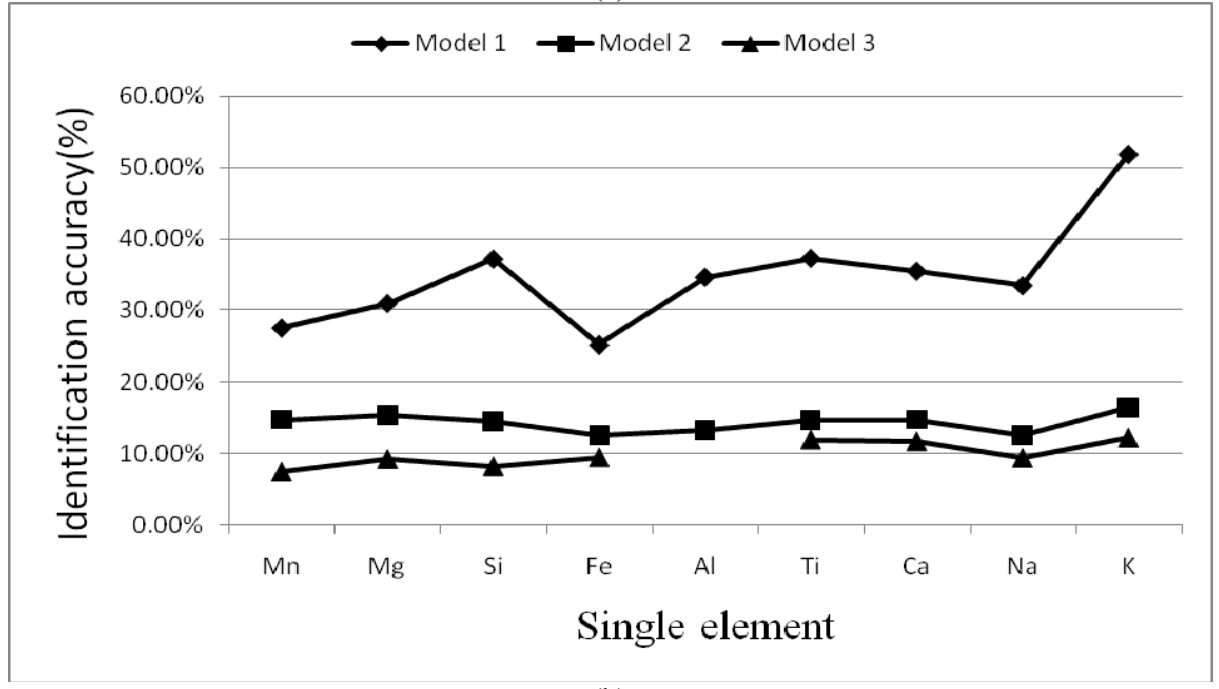

(b)

Fig. 4 The effect of single element: (a) The classification of 6 types of samples; (b) The distinction of 26 samples.

9 elements in the table 1 . That is they had the same sample features, which made the identification accuracies have a certain similarity with the tendency of changing. While the similarity would be enhanced or weakened by the different sample features. In Fig. 4, the maximum and minimum difference of identification accuracy of Model 1 by nearly $25 \%$, but the difference of Model 2 and Model 3 by less than $17 \%$. The reason is that there were more spectral lines and more difference of sample features in Model 1, but they were less in Model 2 and Model 3. Sample features refer to the elements contents, elements distribution, the granularity of the sample, sample interspace, spectral profile, SNR of spectral lines, relative intensity of spectral lines and so on. Furthermore, the mutual influence of these parameters determine the samples features.

4.3 The Effect of the Combination of 8 Elements Chosen from 9 Elements on the Identification Accuracy of BPNN Respectively

Selecting 8 elements from 9 elements to constitute the three characteristic spectra which were input to BPNN to sort or distinguish samples. The effect of combinations of 8 elements on the identification accuracy of BPNN respectively as shown in Fig. 5, 
identification accuracies of the WSLM can be referenced in Table 3.

From Fig. 5 it can be seen, whether for samples classification or distinction, whatever the elements selected, the identification accuracies of Model 1 were much higher than that of Model 2. We can also obtain that BPNN identification accuracies of these two characteristic models had a certain similarity with the tendency of elements changing which was complementary to the tendency of one element as shown in Fig. 4. For example, when 6 types of samples are sorting, if there was only one element as

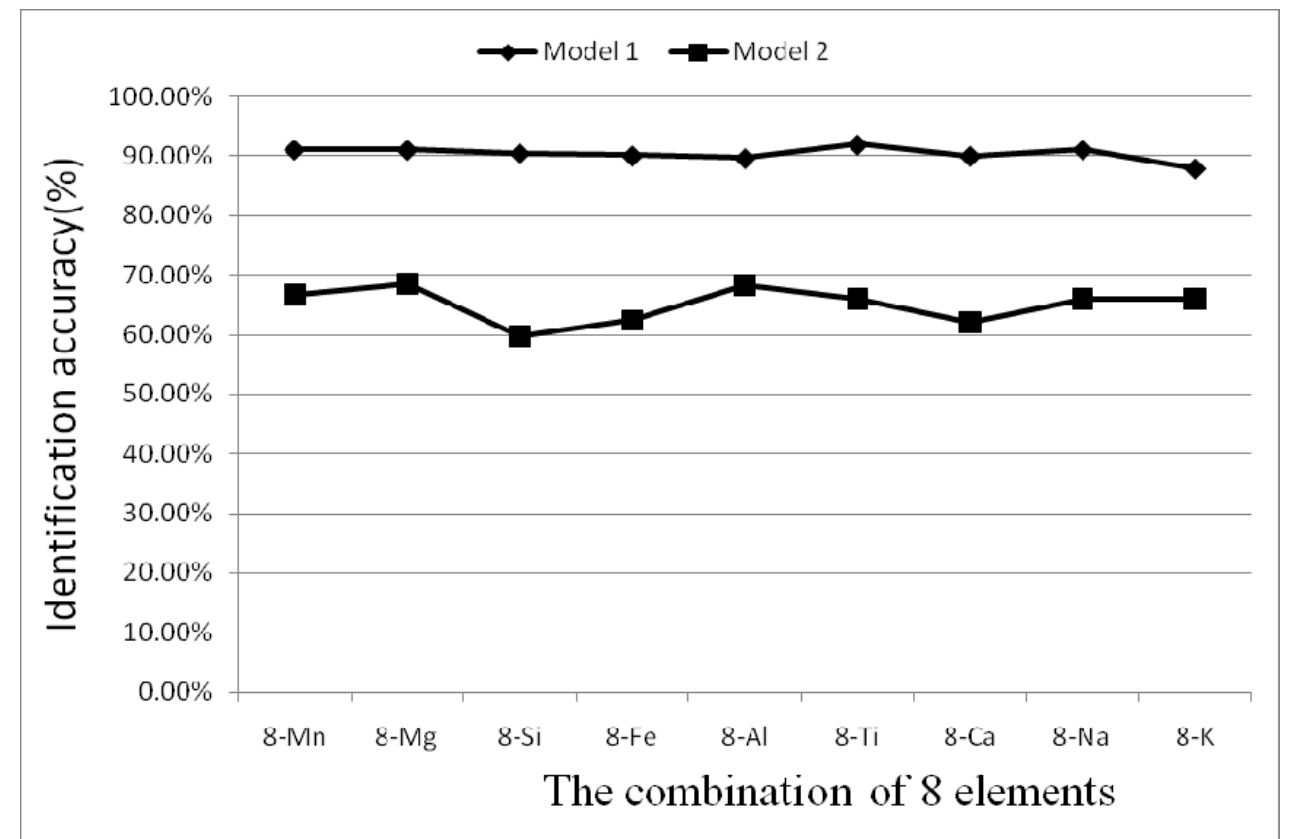

(a)

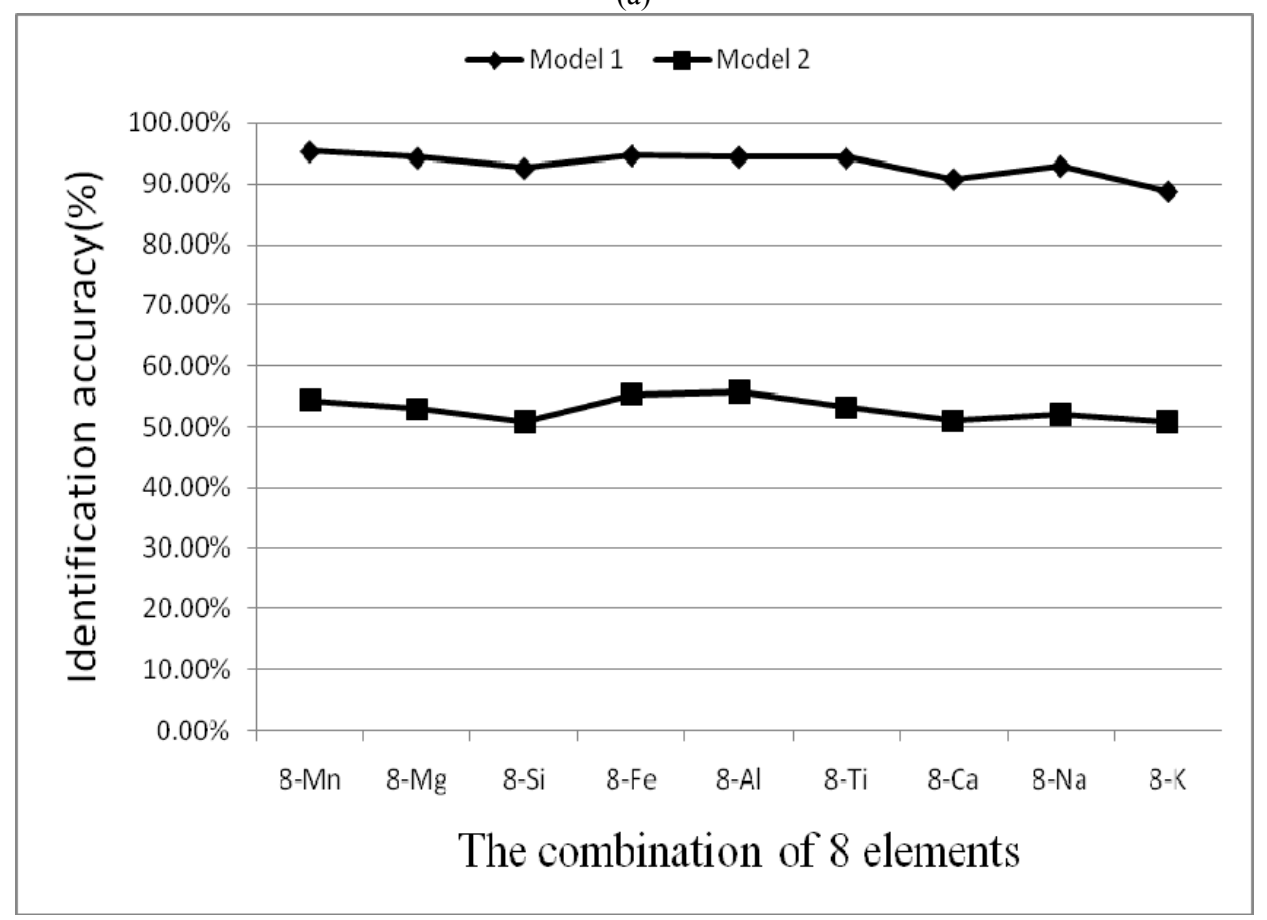

(b)

Fig. 5 The effect of the combination of 8 elements: (a) The classification of 6 types of samples; (b) The distinction of 26 samples. 


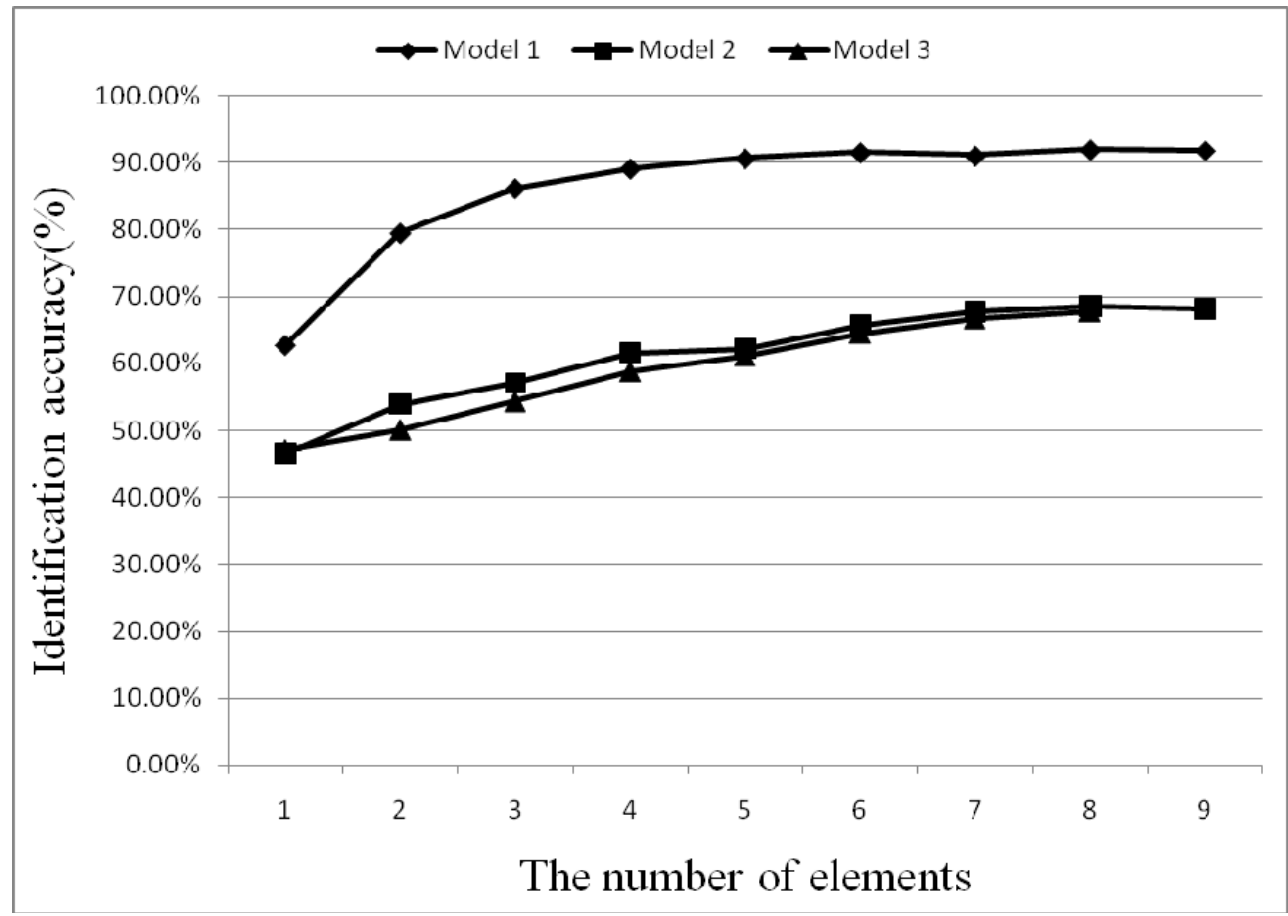

(a)

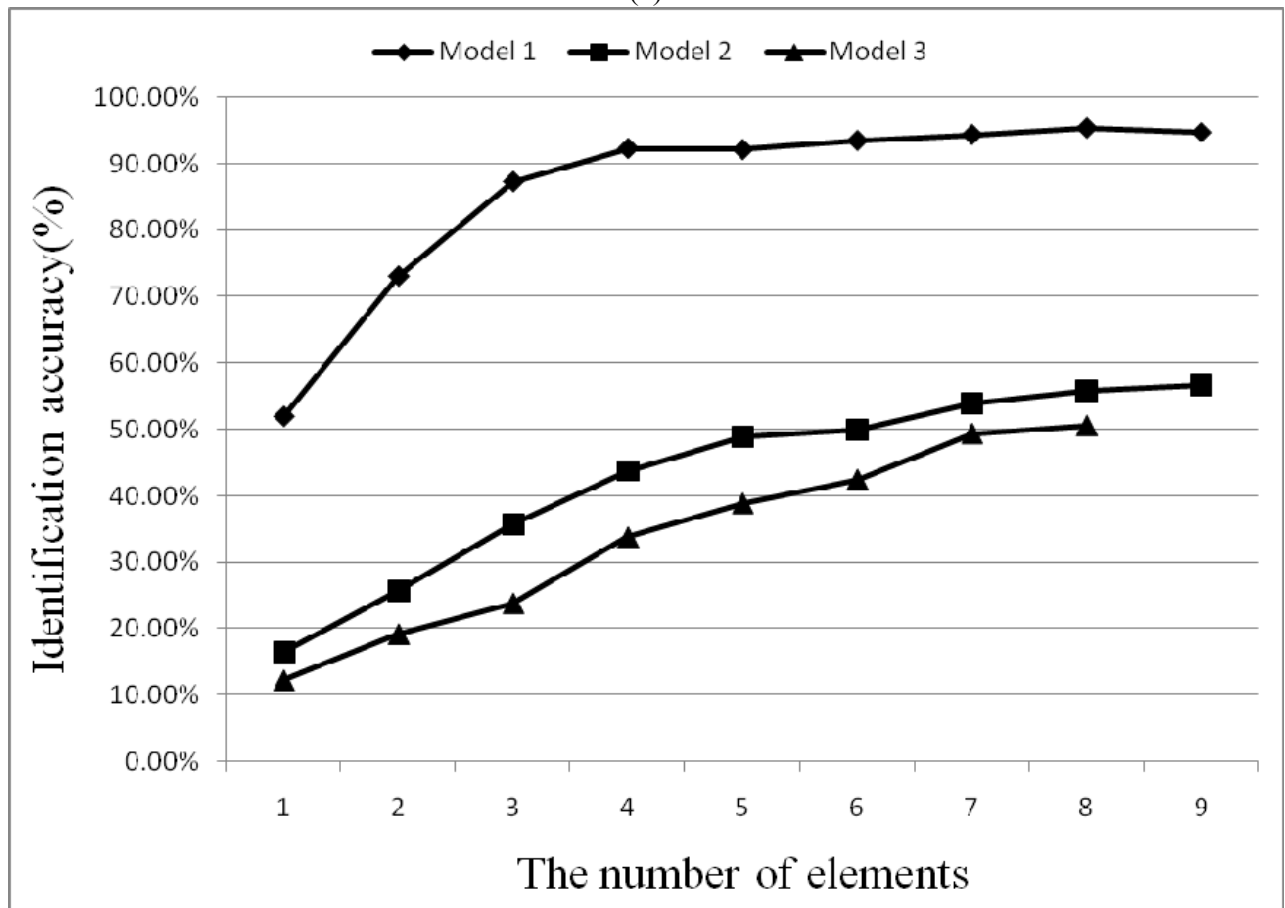

(b)

Fig. 6 The effect of the number of elements: (a) The classification of 6 types of samples; (b) The distinction of 26 samples.

shown in Fig. 4, identification accuracy of Model 1 was on the upward trend of $\mathrm{Na}$ and $\mathrm{K}$, while identification accuracy of Model 2 was on the fall trend of them. But if there was the combination of 8 elements as shown in Fig. 5, the trend of identification accuracy of Model 1 and Model 2 was reverse. This rule can apply to the other elements.

According to Figs. 4 and 5, if identification accuracies of combinations of 8 elements and the remained 1 element were translated into the same 
benchmark, the sum of identification accuracies were almost equal to that of 9 elements combination. But there existed a small deviation of the complementarity. The identification accuracies of 9 elements combination were shown in Fig. 6. The small deviation of complementarity as above-mentioned was caused by the interaction of the sample characteristics of 8 elements and the sample characteristics of 1 remained element. This interaction would increase or decrease the identification accuracy. In Fig. 5, whatever the element was removed, the relative change of the BPNN identification accuracies of 8 remained elements was about $0-10 \%$. That means when the selected elements reached a certain number, 7 or more, the effect of removing a element was not great for the sample classification or sample distinction.

\subsection{The Effect of the Number of elements on the Identification Accuracy of BPNN}

Selecting spectra lines from 26 samples to constitute three characteristic spectra which were input to BPNN to sort or distinguish samples. The effect of the number of elements on the identification accuracy of BPNN as shown in Fig. 6.

In Fig. 6, 9 elements were $\mathrm{Si}, \mathrm{Al}, \mathrm{Fe}, \mathrm{K}, \mathrm{Ca}, \mathrm{Mg}, \mathrm{Na}$, Ti and Mn. 8 elements meant selecting 8 from these 9 elements. 7 elements meant selecting 7 from these 9 elements. We can deduce the rest from this, then 1 element meant selecting 1 from these 9 elements. Those combinations of two or three elements selected from these 9 elements would be a lot of groups. According to the sequence of BPNN identification accuracy of single element in Table 2, we selected elements which correspond to the higher identification accuracy to combine for reducing the groups. For three characteristic models, we selected combinations of elements all in the same way. In Fig. 6, those identification accuracies of BPNN referred to the maximum value of several combinations in three characteristic models. Because Al was the reference element in Model 2, that there remained only 8 elements to combine.

From Fig. 6, it can be seen that identification accuracies of three characteristic models varied with the increasing of selected number of elements and shown a curve of increasing, which was most obvious in Model 1, but they were slightly flat in the other two models and more close to linear increasing. When the number of the selected elements was five, identification accuracy of Model 1 had been as high as $90 \%$, continuing to increase the number of elements for improving the identification accuracy was not obvious. Considering the influence of time consuming as shown in Fig. 7, we selected 5 to 7 elements is more appropriate.

If the difference between types of samples was bigger than the difference between the samples, the identification accuracy of samples classification should be higher than that of samples distinction. In Fig. 6, when classification and distinction of 26 samples, identification accuracies of Model 2 and Model 3 were in line with this regular pattern. But if the selected number of elements was more than three, the identification accuracy of Model 1 was began to break this regular pattern. That may due to the characteristic spectra of each element consisted of 15 spectral lines in Model 1. These lines increased the characteristic difference between samples and samples far larger than the characteristic difference between types and types.

Whatever the number of elements, identification accuracies of Model 1 were much higher than that of the other two models, and identification accuracies of the other two models were close. But identification accuracies of Model 2 were slightly higher than that of Model 3 overall. That was due to Model 2 contains the characteristic information of samples more than that of Model 3. Model 3 was essentially the concentrated characteristics information of Model 2, that we could use fewer elements obtain higher identification accuracy of samples classification in a certain extent, 
but also reduce the identification accuracy of samples distinction. This point can been confirmed in Fig. 4 and Fig. 6.

The effect of the number of elements on the time consuming of BPNN was shown in Fig. 7. From this figure, we can see that with the increasing of the number of elements, the time consuming of program of Model 2 and Model 3 increased slowly even not obviously, but the time consuming of program of Model 1 was almost linear increasing.

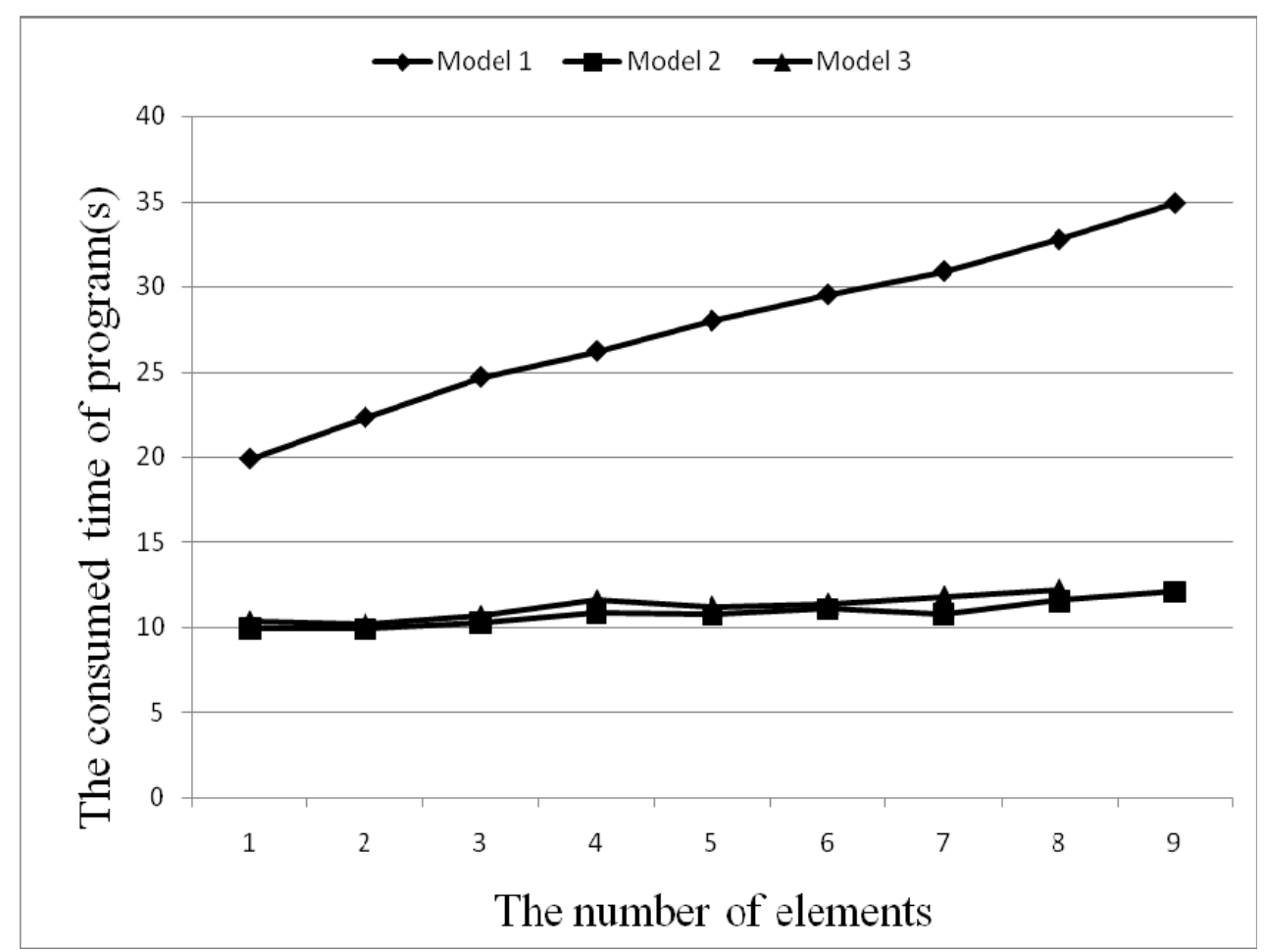

(a)

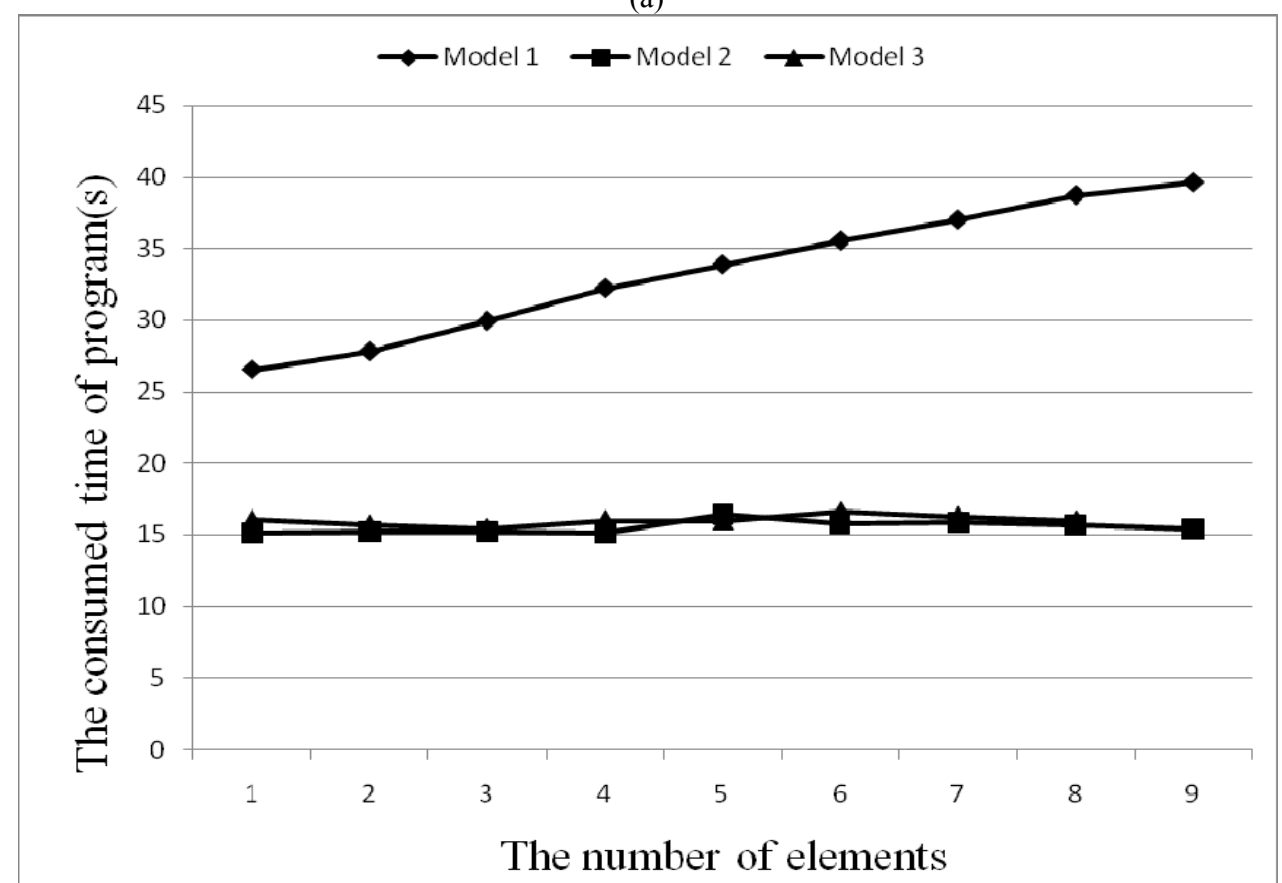

(b)

Fig. 7 The effect for the time consuming : (a) The classification of 6 types of samples; (b) The distinction of 26 samples. 
The data quantity of Model 1 was by 10 times more than that of Model 2 and Model 3, so the time consuming of program of Model 1 was more. With increasing of the number of elements, time consuming also would present an obvious increasing trend. However spectral lines of Model 2 and Model 3 were less, with increasing of the number of elements, the trend of time consuming was not obvious. The time consuming of program was mainly consumed during the training of BPNN. When the amount of data was small, time consuming was not obvious in the training process. On the contrary, it was.

\section{Conclusions}

According to contents of the major elements in 26 samples, we selected spectral lines of the 9 elements from the LIBS spectra to construct the three characteristic models, as follows, the WSLM, the PM and the PRM. Then, the data of these three models were imported into BPNN to sort the samples of 6 types and distinguish 26 samples. The conclusions as follow:

(1) When the BPNN analysis of combination of several elements was carried out, selecting the combination of elements with high identification accuracy of single element BPNN analysis, then the identification accuracy of this combination would be higher.

(2) In the three characteristic models, identification accuracies of the WSLM were much higher than that of the other two models overall.

(3) Considering the time consuming of program, if selecting 5 to 7 elements according to contents of main elements in samples, the identification accuracy of the WSLM for sorting and distinguishing samples had been as high as $90 \%$, continuing to increase the number of elements for improving the identification accuracy was not very obvious.

In the field of LIBS, these conclusions of this paper are of a certain reference for the classification and distinction of unknown samples.

\section{Acknowledgments}

The authors thank the Development of Science and Technology Program of JingZhou (Grant 2015AB35) and The 7th National Undergraduate Innovation and Entrepreneurship training Program of Yangtze University (Grant 2014036) for the financial supports. Thanks are due to professor Wang for providing guidance and help, to Wang ShaoLong for helping us for the pretreatment of samples.

\section{References}

[1] Arab, M., Bidin, N. and Chaudhary, K. et al. 2015. "Characterization of Pollution Indices in Soil Surrounding a Power Plant by Laser Induced Breakdown Spectroscopy. " Analytical Letters 48 (2): 360-70.

[2] Yu, X. D., Li, Y. and Gu, X. F. et al. 2014. "Laser-Induced Breakdown Spectroscopy Application in Environmental Monitoring of Water Quality: a Review (in Chinese)." Environmental Monitoring and Assessment 186 (12) : 8969-80.

[3] Wei, L., Wang, L. Q. and Zhou, T. et al. 2012. "Application and Progress of the Nondestructive Spectral Technology Used in Polychrome Ceramic Relics Analysis (in Chinese)." Spectroscopy and Spectral Analysis 32 (2): 481-5.

[4] Kasem, M. A., Gonzalez, J. J. and Russo, R. E. et al. 2014. "Effect of the Wavelength on Laser Induced Breakdown Spectrometric Analysis of Archaeological Bone.” Spectrochimica Acta Part B: Atomic Spectroscopy 101 (11): 26-31.

[5] Flores, A. P., Sanchez, A. K. F. and Villarreal, A. et al. 2015. "The Potential of Compact Libs System with Multi-Pulse Nd:YAG Laser for Bacteria Identification." Journal of Biomedical Science and Engineering 8 (3): 207-12.

[6] Liu, X. N., Wu, Z. S. and Qiao, Y. J. 2013. "Review on Rapid Evaluation of Product Quality Attributes and Application Prospects in Chinese Materia Medica (in Chinese)." World Chinese Medicine 8 (11): 1269-72.

[7] He, X. W., Huang, L. and Liu, M. H. et al. 2014. "Determination of $\mathrm{Cd}$ in Rice by Laser-Induced Breakdown Spectroscopy (in Chinese)." Applied Laser 34 (1): 72-5.

[8] Multari, R. A., Cremers, D. A. and Dupre, J. A. et al. 2013. "Detection of Biological Contaminants on Foods and Food Surfaces Using Laser-Induced Breakdown Spectroscopy (LIBS)." Journal of Agricultural and Food Chemistry 61 (36): 8687-94. 
[9] Fabrea, C., Cousinb, A. and Wiensb, R. C. et al. 2014. "In Situ Calibration Using Univariate Analyses Based on the Onboard ChemCam Targets: First Identification of Martian Rock and Soil Compositions." Spectrochimica Acta Part B: Atomic Spectroscopy 99 (9): 34-51.

[10] Mangold, N., Forni, O. and Dromart, G. et al. 2015. "Chemical Variations in Yellowknife Bay Formation Sedimentary Rocks Analyzed by ChemCam on Board the Curiosity Rover on Mars." Journal of Geophysical Research: Planets 120 (3): 452-82.

[11] Yu, Q., Ma, X. H. and Wang, R. et al. 2014. "Research on Fast Classification Based on LIBS Technology and Principle Component Analyses (in Chinese)." Spectroscopy and Spectral Analysis 34 (11): 3095-9.

[12] Chen, X. L., Dong, F. Z. and Tao, G. Q. et al. 2013. "Fast Lithology Identification by Laser-Induced Breakdown Spectroscopy (in Chinese)." Chinese Journal of Lasers 40 (12): 1215001.

[13] Zou, X. H., Hao, Z. Q. and Yi, R. X. et al. 2015. "Quantitative Analysis of Soil by Laser-Induced Breakdown Spectroscopy Using Genetic Algorithm-Partial Least Squares (in Chinese)." Chinese Journal of Analytical Chemistry 43 (2): 181-6.

[14] Yu, Y., Hao, Z. Q. and Li, C. M. et al. 2013.
"Identification of Plastics by Laser-Induced Breakdown Spectroscopy Combined with Support Vector Machine Algorithm (in Chinese). " Acta Phys. Sin. 62 (21): 215201.

[15] Wang, C., Jiang, D. H. and Yan, Z. Q. et al. 2014. "Element Analysis Technique Based on Laser-Induced Breakdown Spectroscopy (in Chinese)." Mud Logging Engineering 25 (1): 10-5.

[16] Zhu, Y. X. and Shi, G. R. 2013. "Identification of Lithologic Characteristics of Volcanic Rocks by Support Vector Machine (in Chinese)." Acta Petrolei Sinica 34 (2): 312-22.

[17] Du, Z. H., Meng, F. L. and Li, J. Y. et al. 2012. "Research on the Analytical Line Auto-Selection for Quantitative Analysis of Materials with Laser-Induced Breakdown Spectroscopy." Spectroscopy and Spectral Analysis 32 (4): 876-80.

[18] Sun H. W. 2004. Atomic Spectral Analysis (in Chinese). BeiJing: Higher Education Press, China.

[19] Wang, Q. Q., Huang, Z. W. and Liu, K. et al. 2012. "Classification of Plastics with Laser-Induced Breakdown Spectroscopy Based on Principal Component Analysis and Artificial Neural Network Model (in Chinese)." Spectroscopy and Spectral Analysis 32 (12): 3179-82. 